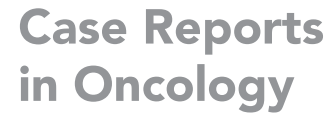

\title{
Epithelioid Hemangioendothelioma and Epithelioid Hemangioma: Pazopanib as a Potential Salvage Therapy
}

\author{
Alexander Yakobson ${ }^{a}$ Wafeek Alguayn $^{b, f} \quad$ Walid Shalata $^{a} \quad$ Daniel Levin ${ }^{c}$ \\ Tawfeek A. Kian ${ }^{d}$ Amir Korngreen ${ }^{d}$ Rachel Gibbs $^{c}$ Mahmuod A. Salah ${ }^{b, f}$

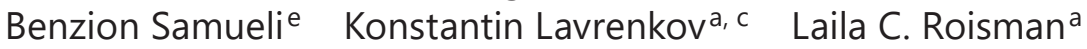 \\ Yulia Dudnik $^{a} \quad$ Nir Peled $^{a}$ Yael Refaely ${ }^{f}$ Waleed Kian ${ }^{a}$ \\ aThe Legacy Heritage Oncology Center \& Dr. Larry Norton Institute, Soroka Medical Center \\ \& Ben-Gurion University, Beer-Sheva, Israel; ${ }^{b}$ Department of Cardiac Surgery, Soroka Medical \\ Center, Beer-Sheva, Israel; ' ${ }^{F}$ aculty of Health Sciences, Ben-Gurion University of the Negev, \\ Beer-Sheva, Israel; dDepartment of Orthopedic Surgery, Soroka Medical Center, Beer-Sheva, \\ Israel; eDepartment of Pathology, Soroka University Medical Center, Beer-Sheva, Israel; \\ fThoracic Surgery Unit, Soroka Medical Center, Beer-Sheva, Israel
}

\section{Keywords}

Rib epithelioid hemangioma $\cdot$ Epithelioid hemangioendothelioma $\cdot$ Pazopanib

\begin{abstract}
Epithelioid hemangioma (EH) and epithelioid hemangioendothelioma (EHE) are both rare vascular tumors. EH tumors are often benign while EHE tumors have moderate malignant potential. Here, we present three unique cases at Soroka Medical Center, two featuring EH of the bone and one presenting EHE of the mediastinum. Each case demonstrates distinct treatment challenges due to the rarity of both diseases and lack of established guidelines. We propose three treatment approaches including pazopanib for salvage therapy of EH of the bone and minimally invasive surgical resection which in these cases lead to complete symptom relief and tumor stabilization upheld over time with close follow-up.

(C) 2021 The Author(s).

Published by S. Karger AG, Basel
\end{abstract}

Alexander Yakobson and Wafeek Alguayn contributed equally to this work. 


\section{Introduction}

Epithelioid hemangioendothelioma (EHE) and epithelioid hemangioma (EH) are a rare group of vascular tumors mainly affecting various soft tissue sites, the lungs, and the liver [1]. As prevalence of these tumors is rare and highly variable and ubiquitous, classifications and guidelines for treatment remain debated [2].

$\mathrm{EH}$ of the bone is relatively rare, locally aggressive but usually benign vascular tumor arising in adults. EH tumors are often characterized by fully formed, capillary-sized lined by histiocytoid or epithelioid endothelial cells and inflammatory infiltrate [3]. Trauma to bone or blood vessels may initiate the pathologic development of EH [4]. Up to 25\% of EH cases may be multifocal, though the majority of tumors are solitary [5, 6]. EH of the bone can be found pervasively in the skeletal system, yet the most commonly involved locations are the femur, tibia, bones of the hand and foot, and bones of the axial skeleton.

Mediastinal endothelial hemangioend othelioma is also a rare, low- to intermediate-grade vascular neoplasm with borderline malignant potential. A novel fusion gene, WWTR1CAMTA1, was recently discovered as being highly specific to EHE [7]. Cases are often identified incidentally on chest imagining with bilateral pulmonary lesions. We present three ultra-rare cases including one case of mediastinal EHE and two cases of bone EH along with treatment recommendations and subsequent follow-up results.

\section{Case 1}

A 49-year-old Bedouin female with a past medical history of tobacco use presented with a painful lesion in right side of the chest wall in May 2019. Chest X-ray showed lytic lesions with rib fracture (Fig. 1A) and a chest CT-scan showed lytic lesion of the seventh rib (Fig. 1B, C). Biopsy of the affected rib showed histological findings and immunostaining results
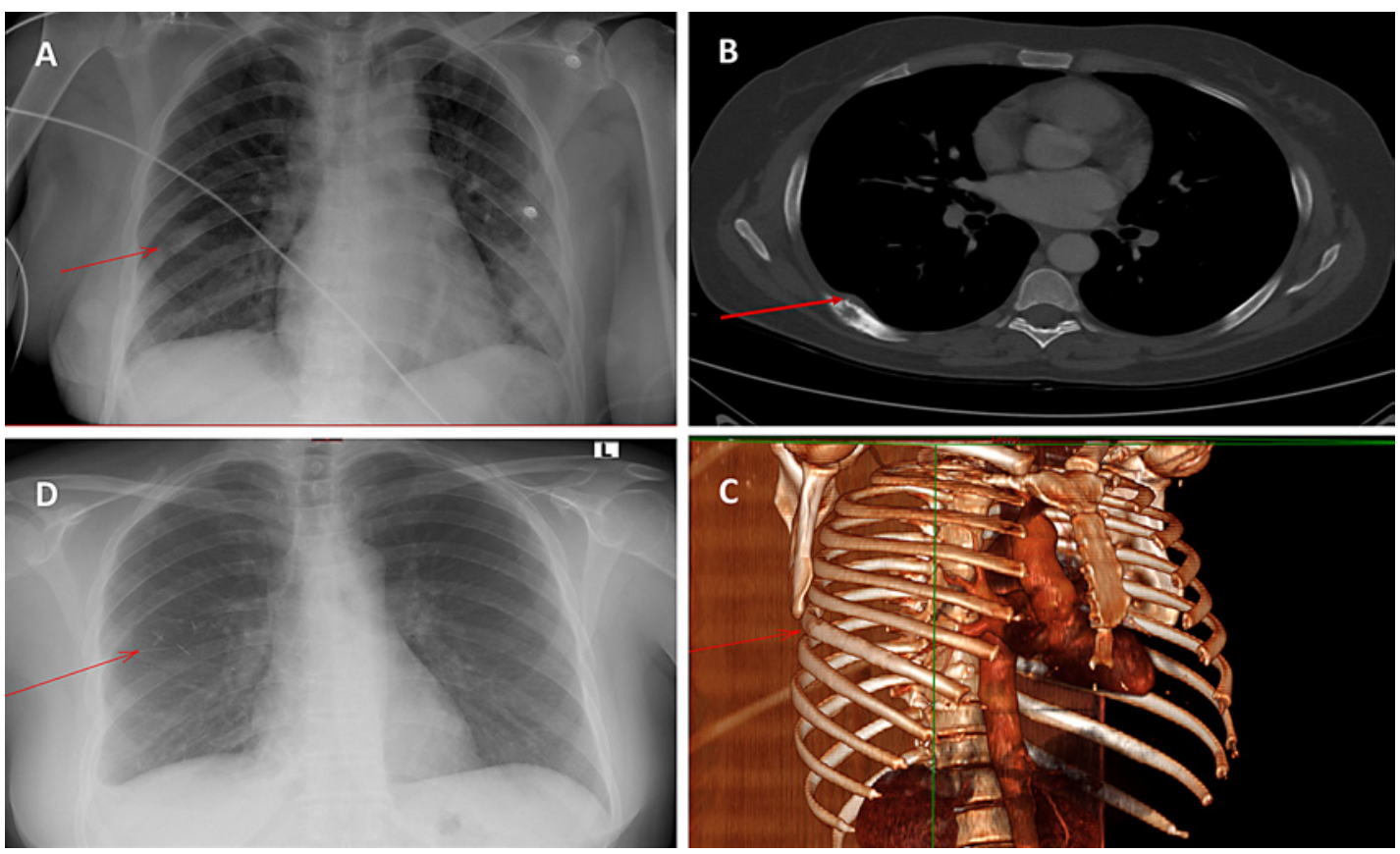

Fig. 1. A Chest X-ray with lytic lesions and fracture of the right seventh rib. B, C Chest CT scan with lytic and sclerotic lesions and deformity of the right seventh rib. D Chest X-ray after right seventh rib resection. 


\section{Case Reports in Oncology}
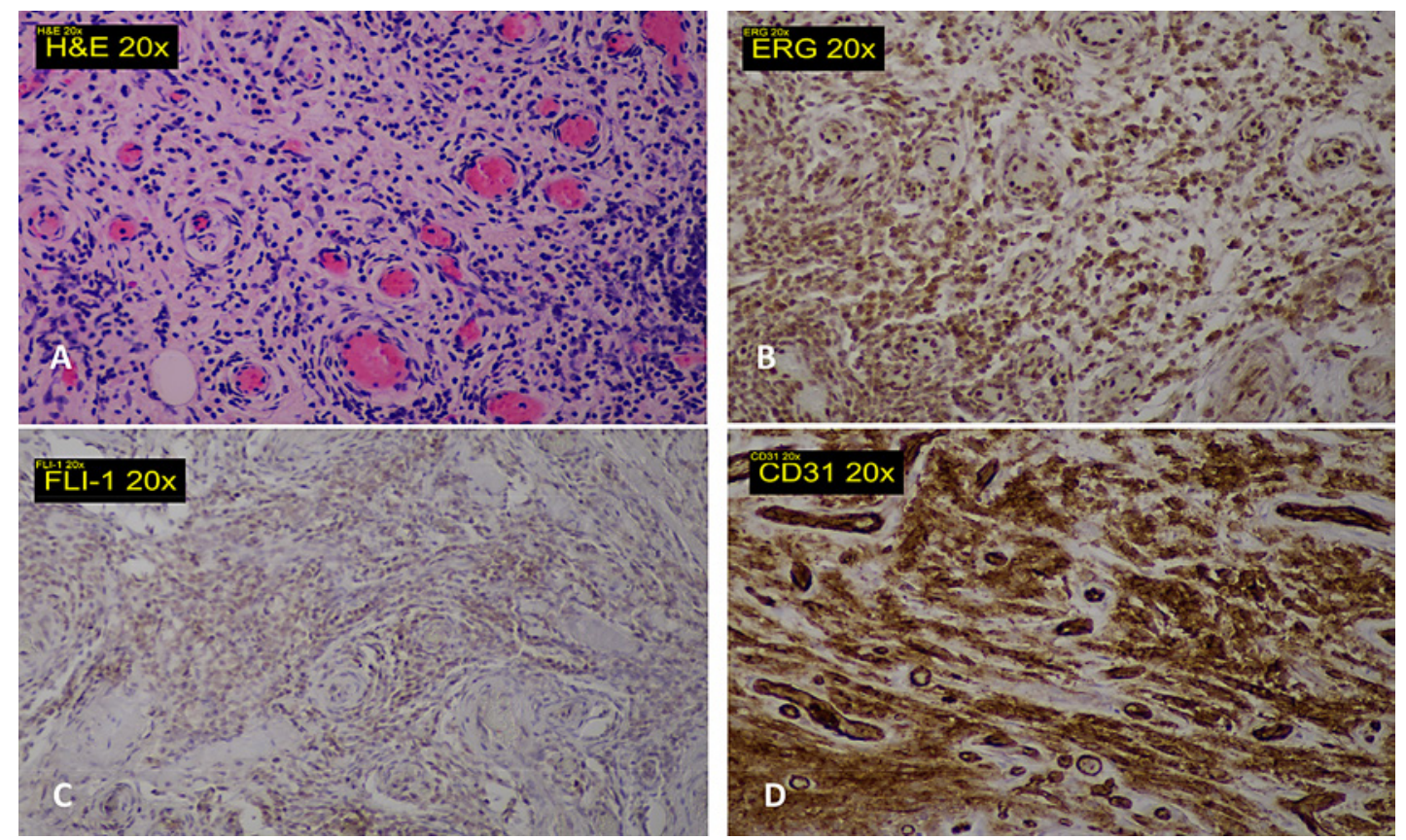

Fig. 2. A H\&E stain showing a proliferation of small, well-formed blood vessels with epithelioid endothelium. In the stroma, there is proliferation of spindle and oval cells without vascular lumen formation. B-D Immunostains for CD31, ERG, and FLI1 (endothelial markers) are positive in both populations of cells (lumenforming and stromal cells). The immunostains also highlight focal cord formation in the stromal population. Original magnification, $\times 20$ (all images).

compatible with angio-lymphoid hyperplasia with eosinophilia and EH (Fig. 2). To evaluate for metastasis and other lesions, a positron emission tomography, computed tomography (PET-CT) was performed showing lytic and sclerotic changes and deformity without significant pathologic uptake in the seventh rib and without distant metastasis.

A right thoracoscopic biopsy using a 5-mm camera was performed taking several samples from the rib. The tumor was seen protruding without penetration into the pleural space. Explorative thoracoscopy showed intact pleura with proper hemostasis and inflation of the lung. The latissimus dorsi muscle and incision were closed without leaving a chest drain.

Biopsy results were compatible with angio-lymphoid hyperplasia with eosinophilia and in this case, EH (Fig. 2). The surgical margins could not be assessed, as the specimen was in fragments. Therefore, excision of the lesion was recommended.

Following the surgery, the patient was readmitted to the hospital several times with severe right chest pain under maximum analgesic treatment. After other chest pain pathologies were excluded, the decision was made to perform a rib resection.

In January 2020, the rib resection was performed through a small incision on the previous surgical scar with two additional incisions for thoracoscopy control and instrument insertion. Tissue was dissected throughout the length of the seventh rib using periosteal elevator and diathermy. The rib was cut posteriorly near the vertebra and anteriorly on cartilage using a Dennis rib cutter. Separation of the rib was done thoracoscopically from the incision on the previous surgical scar, and the rib was extracted completely. Rib approximation was performed, and hemostasis was established before closing the incision and leaving a 28-Fr chest drain tube (Fig. 3A-D). 


\section{Case Reports in Oncology}
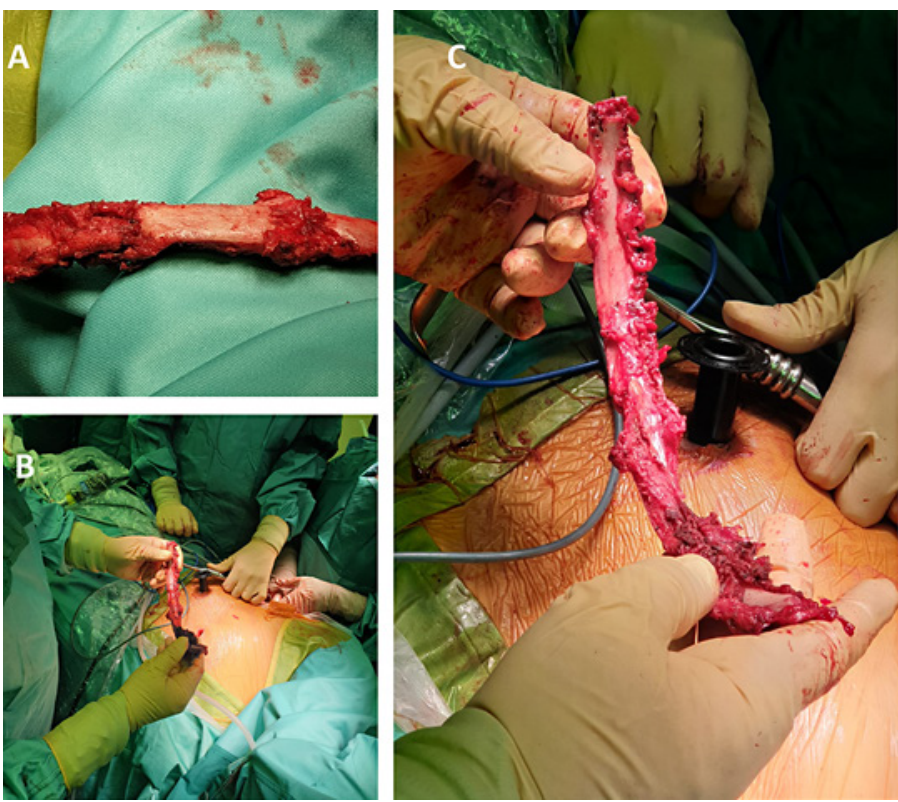

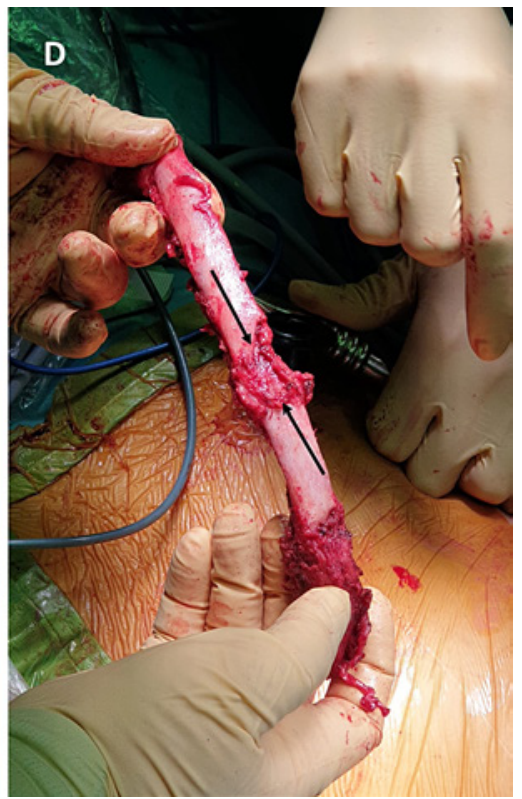

Fig. 3. A-C Surgical figure showing the mass with dimensions of $3 \times 1.5 \mathrm{~cm}$ in the posterior aspect of the right seventh rib. D The distorted rib with two pathologic fractures (black arrows).

Several months after the operation, the patient returned for a follow-up to assess completion of pain relief treatment (Fig. 1D), and she continues with regular hematological follow-up without requiring any additional treatment.

\section{Case 2}

A 66-year-old Russian female with a history of hypertension, dyslipidemia, and diabetes mellitus, was incidentally found to have a $7.5 \mathrm{~cm}$ in diameter mediastinal space occupying lesion (SOL) on a CT-scan in 2015 (Fig. 4A). A CT-guided biopsy was performed demonstrating solitary fibrous tumor, and it was decided to continue with close follow-up. The patient was stable until October 2017 when she was referred to our center due to enlargement of the SOL to $9 \mathrm{~cm}$ in diameter (Fig. 4B). A PET-CT scan was done showing enlargement of the SOL as well as metastasis to the lungs bilaterally, liver and sternal bone (Fig. 4C).

In November 2017, a thoracoscopic biopsy was performed of the mediastinal mass and lung nodules. A highly vascular mass was seen encompassing the phrenic nerve; therefore, further dissection or removal of the tumor was not attempted. The pathologic biopsy result showed EHE (Fig. 5). Genetic profiling results found a missense mutation in AXL V491M in exon 11 with mutation fraction of $47 \%$, and no other amplifications or translocations were identified. The patient started a combination chemotherapy of carboplatin plus paclitaxel and bevacizumab every 3 weeks. In April 2018 after four treatment cycles, a PET-CT showed stable disease. The patient was given bevacizumab for continued single maintenance therapy. After the third cycle of bevacizumab, the patient underwent another PET-CT showing yet again stable disease (Fig. 4D). Due to these promising results, we decided to stop bevacizumab maintenance therapy and continue with close follow-up with PET-CT. In March 2019, 


\section{Case Reports in Oncology}

\begin{tabular}{l|l}
\hline Case Rep Oncol 2021;14:309-317 \\
\hline DOI: 10.1159/000510806 & $\begin{array}{l}\text { @ 2021 The Author(s). Published by S. Karger AG, Basel } \\
\text { www.karger.com/cro }\end{array}$ \\
\hline
\end{tabular}

Yakobson et al.: Pazopanib as Salvage Therapy for Epithelioid Hemangioendothelioma and Hemangioma
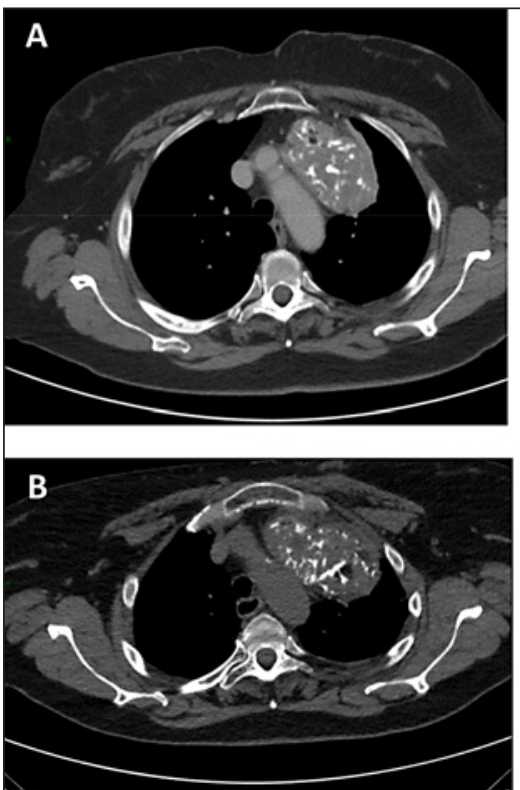

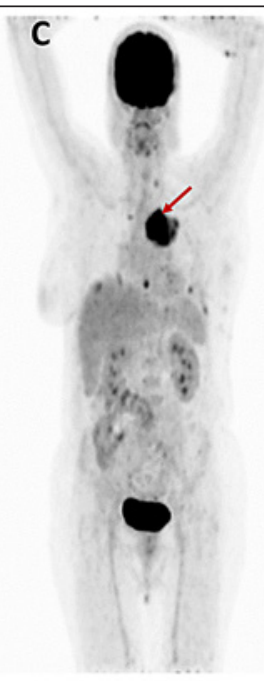

November 2017

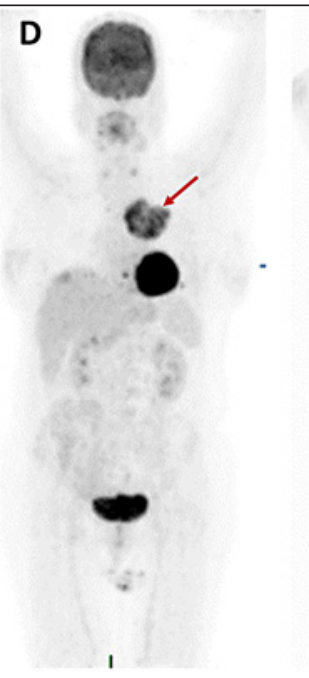

April 2018

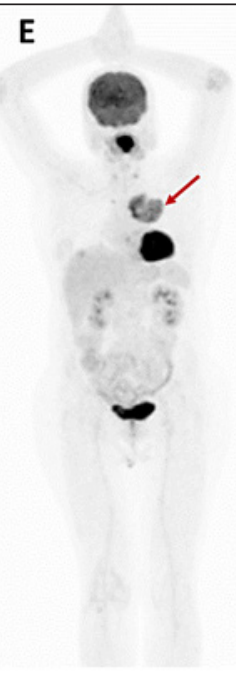

March 2019

Fig. 4. A Chest CT-scan showed a mediastinal space-occupying lesion with $7.5 \mathrm{~cm}$ in diameter. B Enlargement of the mediastinal space-occupying lesion to $9 \mathrm{~cm}$ in diameter. C-E Heterogeneous mass in the left mediastinum measuring $84 \times 50 \mathrm{~mm}$ with hyperdense, gross calcifications. Multiple lung nodules bilaterally, the largest measuring $13 \mathrm{~mm}$ in the left lower lobe. Hypodense lesion in segment 7/8 of liver up to $21 \mathrm{~mm}$. Lytic lesions in the vertebral body at the level of D8. All above lesions had high F18 FDG uptake and suspected as metastasis from the mediastinal mass.
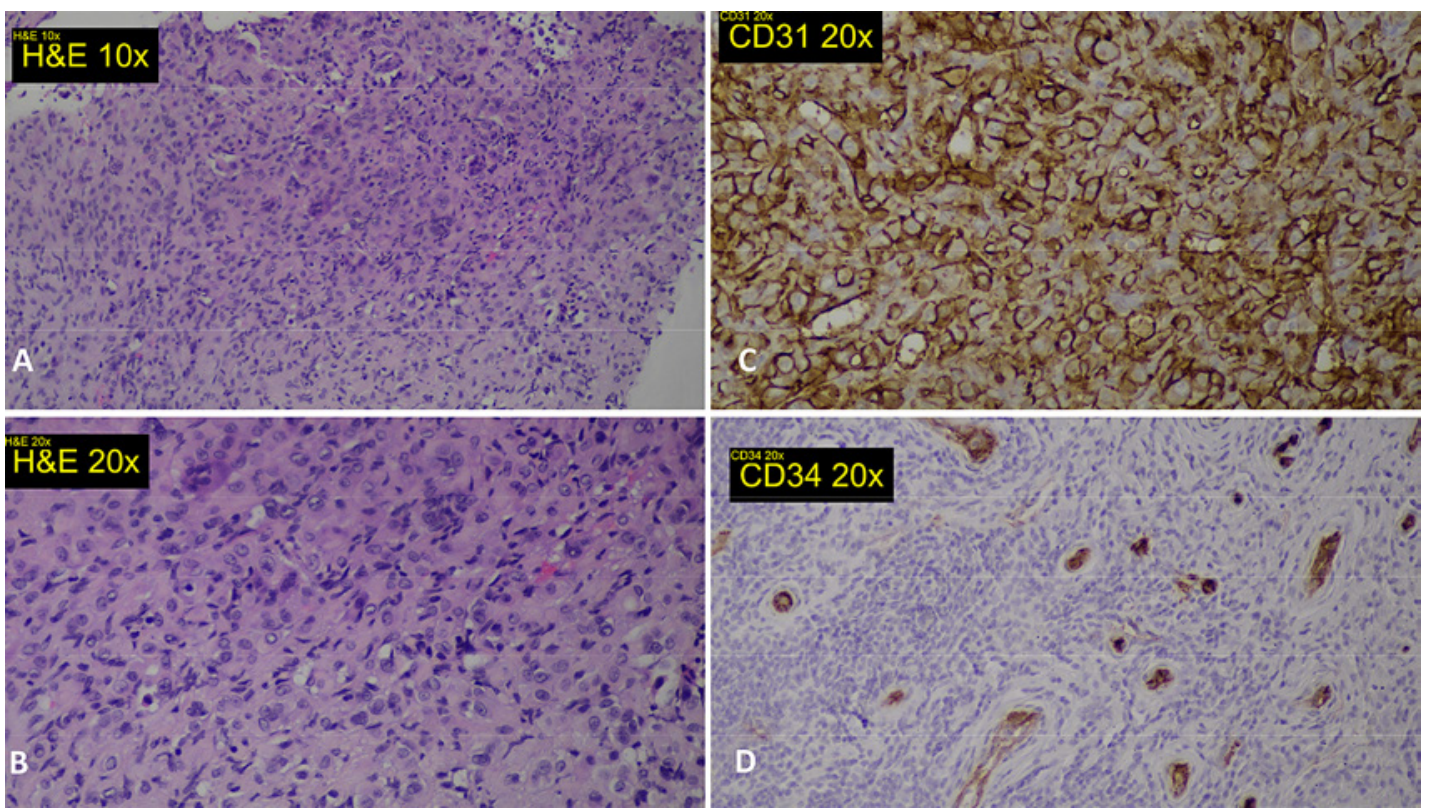

Fig. 5. A, B Tumor composed of medium size, round-to-oval epithelioid cells with a myxoid matrix resembling cartilage. Numerous multinucleated giant cells are also present. Original magnification, $\times 10$ and $\times 20$. C, D Immunostain for CD31 is positive. The remaining immunoprofile showed strong reactivity for other vascular markers (CD34, Factor VIII, and FLI-1) as well as some staining for CK7 and CD10. Original magnification, $\times 20$. 

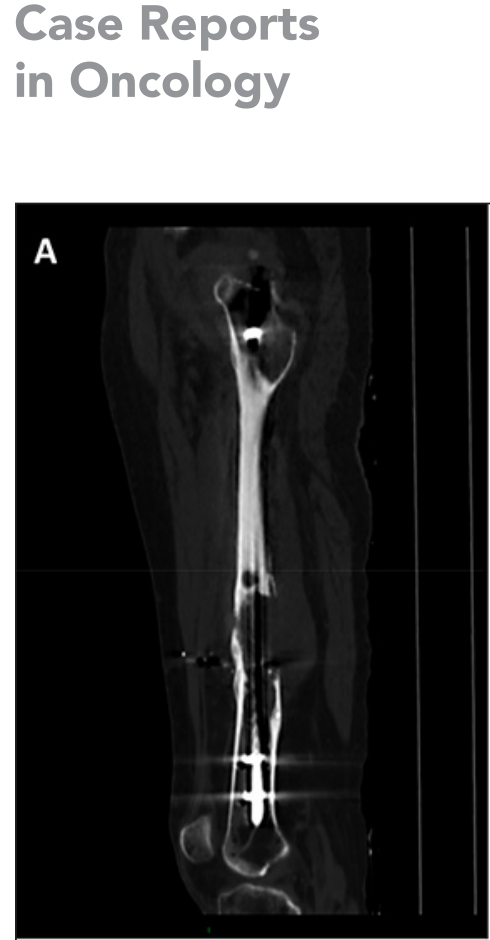

October 2019

\begin{tabular}{l|l}
\hline Case Rep Oncol 2021;14:309-317 \\
\hline DOI: 10.1159/000510806 & $\begin{array}{l}\text { @ 2021 The Author(s). Published by S. Karger AG, Basel } \\
\text { www.karger.com/cro }\end{array}$ \\
\hline
\end{tabular}

Yakobson et al.: Pazopanib as Salvage Therapy for Epithelioid Hemangioendothelioma and Hemangioma

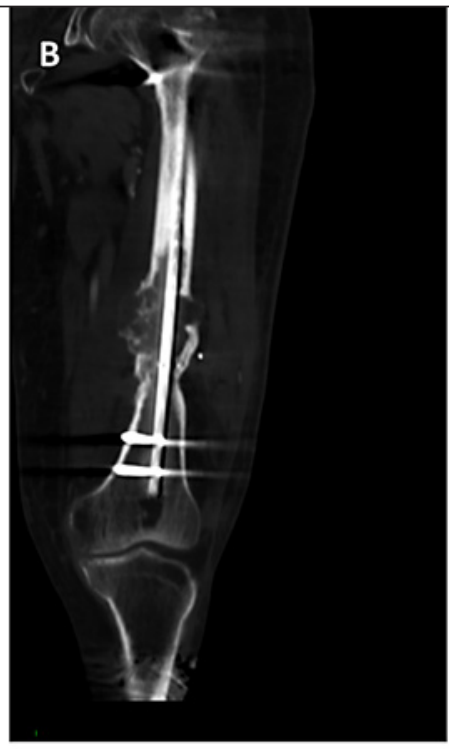

December 2019

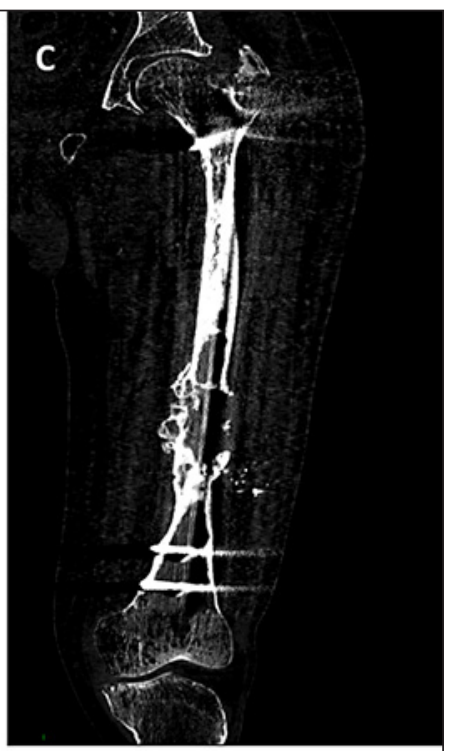

May 2020

Fig. 6. A Cortical destruction of the posterolateral aspect of midshaft femoral bone with additional soft tissue component. B Enlargement of the primary tumor after local radiotherapy. C Soft tissue component decreases in size and bone become more sclerotic following biological therapy.

follow-up PET-CT still demonstrated stable disease, and the decision was made to continue close follow-up with PET-CT every 4 months (Fig. 4E). The patient's disease remains stable with no enlargement or metastasis in May 2020.

\section{Case 3}

A 76-year-old Bedouin man with a history ischemic heart disease, atrial fibrillation, aortic and tricuspid valve disease, hypertension and previous gunshot wound to the left thigh in 1993 treated with intramedullary nailing presented to the orthopedic department at Soroka Medical Center in September 2019 with left hip and thigh pain. The pain had been severe and progressive for several months and accompanied with 20-kg weight loss. Physical examination demonstrated severe thigh swelling with tenderness to palpation throughout the leg and especially above the left knee joint, inability to bear weight, normal strength and without neurovascular abnormalities. He was given analgesic treatment and admitted for further workup.

Lower limb CT-scan revealed a large lytic area in the left femoral shaft cortex with multiple soft tissue compartment involvement (Fig. 6A). A bone biopsy was performed showing a low-grade malignant EH. No bacteria were grown from culture of the biopsy. Thoracic and abdominal CT-scan also revealed no evidence of distant metastasis. The patient was offered urgent surgical amputation of the left leg and pelvis (hemipelvectomy) due to his severe presentation with tumor involvement in multiple compartments; however, the patient refused surgical intervention. He was then discharged and referred to the Oncology Department for follow-up care while a multidisciplinary oncologic team reviewed his case. 
In October 2019, the oncology team decided to proceed with palliative radiation therapy. The first radiation therapy session took place in November 2019 at a total dose of 45 Gy for 15 fractions. The patient experienced significant clinical improvement with reduction of pain severity and disappearance of tight swelling; however, a CT-scan for restaging in December 2019 revealed enlargement of the primary tumor (Fig. 6B). Due to local disease progression, we decided to start with pazopanib $800 \mathrm{mg}$ once daily. The biologic treatment was well tolerated by the patient resulting in further reduction of pain and disability, and he remains with stable disease to date (May 2020) (Fig. 6C).

\section{Discussion}

Due to the rarity of EHE and EH, consensus on treatment and management strategies is poor. We present one case of mediastinal EHE and two cases of EH of the bone, one localized and one metastatic, at Soroka Medical Center in which all three patients were treated with a different approach and reached stable disease with complete symptomatic relief.

The surgical and oncologic guidelines to the treatment EH of the rib are not well established. Prior case reports of rib EH showed successful resection with thoracotomy approach $[8,9]$. With better recovery and cosmetic outcomes, we uphold thoracoscopic surgical rib resection over thoracotomy when surgery with clear margins is achievable as demonstrated in case 1. Early management also improves patients' quality of life and significantly reduces hospital readmissions.

Primary EHE can be found in the liver, soft tissue, and lung [10]. The presentation is often non-specific with $50-75 \%$ of cases being asymptomatic and presenting incidentally on radiographic imaging [11]. The treatment protocol for this type of neoplasm is unclear, but several case reports have shown achievement of stable disease on combination chemotherapy (carboplatin and paclitaxel) with bevacizumab [12]. Our patient in case 2 exemplifies control of metastatic disease under combination chemotherapy along with bevacizumab followed by single agent bevacizumab treatment, and the patient remained stable for more than 2 years to date after stopping bevacizumab.

While surgical resection remains the gold-standard treatment for localized EH of the bone, our patient in case 3 refused surgical intervention leading us to consider palliative radiation therapy followed by pazopanib treatment. Pazopanib is a second-generation multitargeted tyrosine-kinase inhibitor with strong affinity against VEGFR-1/2/3 and with lower affinity toward PDGFR-alpha/beta, FGFR-1/2 and stem cell factor receptor (c-KitR) [13]. In previous trials, pazopanib has been approved as second-line therapy for advanced soft tissue sarcoma while showing limited activity in vascular sarcomas [14]. However, in a retrospective study Kollar et al. [15] showed promising activity for pazopanib against EHE [15]. In a case report Bally et al. [16] demonstrated long-term tumor control with pazopanib in metastatic EHE of the liver. Based on these findings and due to the aggressive nature of the tumor, we decided to add pazopanib for maintenance therapy which led to the achievement of stable disease for 6 months to date. To our knowledge, this is the first reported case showing promising results with pazopanib against locally aggressive $\mathrm{EH}$ of the bone.

\section{Conclusion}

Based on our experience at Soroka Medical Center, we suggest initial monitoring of patients with EH and EHE with interval CT scans to observe disease behavior. If initial imaging demonstrates slow progression of $\mathrm{EH}$ in the bone without evidence of metastasis, minimally

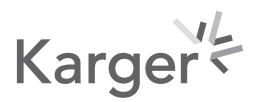


invasive surgery is recommended, and adjuvant radiotherapy may be considered. If the surgical intervention is not possible, palliative radiotherapy followed by target therapy with pazopanib, sorafenib or sunitinib can be offered. For patients with metastatic, slowly progressive EHE, systemic chemotherapy and biological therapy may be initially replaced by close monitoring with CT-scan. Furthermore, in more aggressive disease treatmentapproaches similar to that of angiosarcoma may be considered, as they belong to the same vascular tumor group. Further research is needed to establish the optimal treatment regimens for these ultra-rare diseases.

\section{Acknowledgments}

We would like to thank the patients and the patient families for their cooperation during treatment, and all three patients gave informed consent to be included in this article.

\section{Statement of Ethics}

All subjects have given their written informed consent to publish their case (including publication of images).

\section{Conflict of Interest Statement}

All the authors have no conflicts of interest to declare.

\section{Funding Sources}

None.

\section{Author Contributions}

Conception and design: A.Y., W.A., W.K. Manuscript writing: all authors. Final approval of manuscript: ALL authors.

\section{References}

1 Fletcher CDM, Unni KK, Mertens F, editors. World Health Organization Classification of Tumours. Pathology and Genetics of Tumours of Soft Tissue and Bone. Lyon: IARC Press; 2002. http://www.sciepub.com/ reference/92481

2 Errani C, Zhang L, Panicek DM, Healey JH, Antonescu CR. Epithelioid hemangioma of bone and soft tissue: a reappraisal of a controversial entity. Clin Orthop Relat Res. 2012;470(5):1498-506.

3 Wells GC, Whimster IW. Subcutaneous angiolymphoid hyperplasia with eosinophilia. Br J Dermatol. 1969; 81(1):1-15.

4 Fetsch JF, Weiss SW. Observations concerning the pathogenesis of epithelioid hemangioma (angiolymphoid hyperplasia). Mod Pathol. 1991 Jul;4(4):449-55.

5 O'Connell JX, Nielsen GP, Rosenberg AE. Epithelioid vascular tumors of bone: a review and proposal of a classification scheme. Adv Anat Pathol. 2001;8:74-82.

6 Sung MS, Kim YS, Resnick D. Epithelioid hemangioma of bone. Skeletal Radiol. 2000;29(9):530-4. 
7 Errani C, Zhang L, Sung YS, Hajdu M, Singer S, Maki RG, et al. A novel WWTR1-CAMTA1 gene fusion is a consistent abnormality in epithelioid hemangioendothelioma of different anatomic sites. Genes Chromosomes Cancer. 2011;50(8):644-53.

8 Olfa Ismail MM. Epithelioid hemangioendothelioma of the rib: a rare tumour with a confusing localization. J Clin Exp Pathol. 2014;4(2):1-3.

9 Chirieac LR, Rice DC, Raymond AK. Epithelioid hemangioendothelioma in a patient with unusual involvement of the rib and intercostal lymph nodes. J Thorac Cardiovasc Surg. 2006;132(6):1488-9.

10 HR M, KG I, ZD G. Epithelioid hemangioendothelioma of the liver: a clinicopathologic study of 137 cases. Cancer. 1999;85.

11 Patrini D, Scolamiero L, Khiroya R, Lawrence D, Borg E, Hayward M, et al. Mediastinal hemangioendothelioma: case report and review of the literature. Respir Med Case Rep. 2017;22:19-23.

12 Gaur S, Torabi A, O'Neill TJ. Activity of angiogenesis inhibitors in metastatic epithelioid hemangioendothelioma: a case report. Cancer Biol Med. 2012;9(2):133-6.

13 Van Der Graaf WT, Blay JY, Chawla SP, Kim DW, Bui-Nguyen B, Casali PG, et al. Pazopanib for metastatic softtissue sarcoma (PALETTE): a randomised, double-blind, placebo-controlled phase 3 trial. Lancet. 2012; 379(9829):1879-86.

14 Lee ATJ, Jones RL, Huang PH. Pazopanib in advanced soft tissue sarcomas. Sig Transduct Target Ther. 2019; $4(1): 1-10$.

15 Kollár A, Jones RL, Stacchiotti S, Gelderblom H, Guida M, Grignani G, et al. Pazopanib in advanced vascular sarcomas: an EORTC Soft Tissue and Bone Sarcoma Group (STBSG) retrospective analysis. Acta Oncol. 2017; 56(1):88-92.

16 Bally O, Tassy L, Richioud B, Decouvelaere AV, Blay JY, Derbel O. Eight years tumor control with pazopanib for a metastatic resistant epithelioid hemangioendothelioma. Clin Sarcoma Res. 2015;5:12. 